\title{
Development of Flexible Drilling Jig for Wing-Fuselage Connection
}

\author{
Junshan $\mathrm{Hu}^{1}$, Kaifu Zhang ${ }^{*}, 1$, Hui Cheng ${ }^{1}$ and Siren $\mathrm{Liu}^{2}$ \\ ${ }^{I}$ The Key Laboratory of Contemporary Design and Integrated Manufacturing Technology, Ministry of Education, \\ Northwestern Polytechnical University, Xi'an 710072, China \\ ${ }^{2}$ Shanghai Aircraft Manufacturing Co., Ltd., Shanghai, 200436, China
}

\begin{abstract}
Drilling jigs are used frequently to provide precise location of a hole pattern for mating parts and assemblies in aircraft fabricating process for their accuracy and simplicity. Due to the different product batches and manufacturing process, the components which will be assembled together possess distinct geometry properties or reserve different edge margins, thus the location and the size of connecting holes are not identical. In this case, the pilot holes in the template need to be aligned for every batch of aircraft and the drill templates with fixed guide holes in them are no longer suitable to the situation. This paper proposes a kind of flexible drill template system which is adjustable for precisely locating and drilling holes on a curved surface of the wing-skin during the period of wing-fuselage connection. The jig system possesses a board of curved surface with continually changing curvature which is designed to fit the wing-skin workpiece. The flexible adjustable modules on the board can be adjusted in lateral and spanwise directions in a range of $\pm 10 \mathrm{~mm}$ with a minimum scale of $0.02 \mathrm{~mm}$. The analysis of systematic error and deformation due to operation are also carried out using theoretical model and FE method respectively to verify the validation of the flexible drilling template solution.
\end{abstract}

Keywords: Adjustable, aircraft assembly, deformation, drilling jig, systematic error.

\section{INTRODUCTION}

In aircraft manufacturing process, the assembly is a complicated and vital process where many parts with the characteristic of large size, small thickness, complex shape, poor stability will be mated together and most of them are connected by mechanically fastened joints. In this situation, numerous holes need to be made in mating interfaces for joint. These various kinds of holes, drilled for rivet or bolt connection on different materials, are one of the most crucial factors to the assembly quality and reliability, which directly affect the bearing capacity and service life of aircrafts. Fortunately, the drilling jig provides an effective approach to ensuring assembly quality and shortening assembly cycle.

The drilling jig, also known as drilling template, is a kind of gripper widely used to precisely locate and uprightly guide drilling tools to the designed area to make connecting holes in the fabrication and assembly of aircrafts, from small structural elements to major components such as fuselage and airfoil [1]. A typical drilling jig consists of a flat metal plate with a hole pattern in it. The template is clamped to a part and holes are drilled into the part using the template hole as a guide. When the template is removed, the part has a matching set of holes [2]. The advantage of using a drill template is that the position of tolerance of the holes in the pattern is extremely small. The jig helps to find the right drilling points in the workpiece, to keep connecting holes normal to the mating interfaces and to prevent deviation

*Address correspondence to this author at the Ministry of Education Key Lab of Contemporary Design and Integrated Manufacturing Technology, Northwestern Polytechnical University, Xi'an, 710072, China;

Tel: +86 29 88493303; E-mail: zhangkf@nwpu.edu.cn caused by tool vibration. Mating parts with holes drilled using the same template will fit together precisely.

Although many drilling tasks in assembly can be accomplished by auto machines such as CNC drilling system nowadays, there are some situations where a drilling template is more preferable than automatic tools: 1) the mating parts are supplied by different vendors or fabricated in different places, 2) the components have such complex structures that specialized drilling machines cost too much and can't achieve the required accuracy within the given tolerance, 3) the operating space is too narrow to accommodate automatic equipment, and 4) hole patterns are tightly tolerant to themselves but not to the mating parts. In these cases, a humble cordless drill used with a drilling template can perform the task just as well as the costly CNC milling machines when properly used.

Due to the actual demand, many scholars have done a lot of researches on the issue. Robinson [3] systematically gave several practical tips and useful principles gathered from technicians and engineers on the template design considerations. Jayaweera [4] described the development and test of flexible tooling to provide clamping and support for drilling wing box ribs posts within a restricted environment. Wiemann [5] introduced a flexible template for drill units which can be pinned into piloted datum holes, allowing the jig to find the form of the work piece. Lostlen [6] declared an adjustable drill jig aimed at kitchen cabinet components, and it can be readily assembled to form various modifications of the template for different applications. Most of the research centers on the principle and procedure of designing a template and the solutions are specialized for general drilling tasks of regular parts such as flat plane, while few attentions 


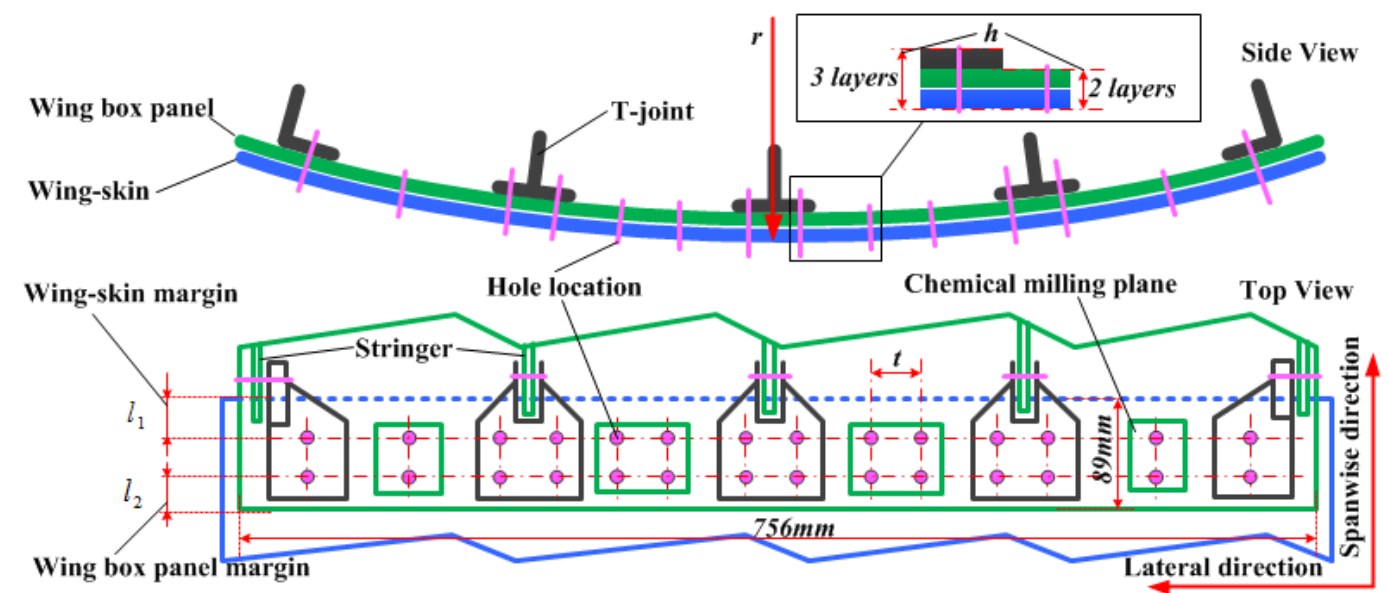

Fig. (1). Configurations of assembly problems.

are paid to the irregular curved surface on which the accuracy of drilling is difficult to secure.

This paper proposes a flexible adjustable drilling jig system for locating and drilling connecting holes on the curved wing-skin profile in the wing-fuselage connection. The jig system contains two jigs to alternatively accommodate 28 holes in a cramped workpiece. The flexible adjustable modules on the jig designed in charge of 9 groups of holes can be adjusted in lateral and spanwise directions to adapt to the changing positions of holes in mating parts manufactured by different vendors. The drilling template in each modulus enables the drilling of holes that are normal to an exposed surface accurately. The technical specification of the drilling jig system is introduced in detail to associate the users.

\section{FLEXIBLE DRILLING JIG SOLUTION FOR ASSEMBLY PROBLEM}

\subsection{Configurations of Assembly Problem}

Aircrafts are made up of massive components, most of which are fixed together by mechanically fastened joints, so drilling on the mating interfaces is an inevitable task in aircraft assembly. Many problems have to be faced with on drilling issue, just as the problem occurs in the wing-fuselage connecting process.

The relative assembling position of wing box and wing during the docking state of wing-fuselage are illustrated in Fig. (1), where the mating interfaces of the wing box panel and the wing-skin are overlapped and clamped to be drilled for connection. There are two kinds of connection types in the mating area. The thicker one is a stackup of wing-skin and wing box. The hole will be made through chemical milling planes on the wing box. The other kind is a threelayer structure piled by wing-skin, wing box panel and Tjoint pile in sequence. The lug of T-joint is connected with the stringer of the wing box while the plate portion of $\mathrm{T}$-joint is in the piling area and waiting to be drilled on it.

The drilling workspace is about $756 \times 89 \mathrm{~mm}^{2}$ where there are two rows of 28 holes to be drilled. The wing box panel together with the wing-skin is ruled surface with gradual radius and their straight-line generatrix coincides with the spanwise direction, so does the row line. Since components of wing box panel and wing-skin are fabricated in different factories, the pre-reserved edge margins $l_{1}$ and $l_{2}$ are not identical due to different batches provided by distinct vendors. Although mechanically fastened joints are effective and reliable, the interaction of hole and bolt easily affects the edge of the panel and the wing-skin without sufficient margins, leading to stress concentration in the juncture, which will cause damage to the material and decrease the capability of the connecting structures. Hence enough margins should be left around the connecting holes in the joint zone to secure the connection strength.

As mentioned earlier, the dimension of margins changes along with the product batches of components, so the hole locations are not fixed as the $3 \mathrm{D}$ model presents. A drilling template with fixed hole patterns would not solve the problem. The hole should be flexibly aligned to the optimal position according to the given edge margin. At the same time the permitted tolerance should be secured. A flexible adjustable drilling jig system is introduced in the following text.

\subsection{Flexible Drilling Jig Solution}

According to the configuration of wing-fuselage assembly, there are two types of stackups to be drilled, namely Type A: the stackup of wing-skin and wing box panel, and Type B: the Type A with a T-joint. Both of two types are explicitly shown in the side view in Fig. (1). Considering the relative distance among the holes and the parts they are located in (chemical milling plane of the panel or the plane of T-joint), each type is divided into several groups (Type A has four groups while Type B possesses five), containing two or four holes. The division is presented in Fig. (3).

Since the workpiece is a narrow and limited area with 28 holes whose positions need to be adjusted in lateral and spanwise directions, two drilling jigs are designed alternatively covering all the holes in drilling workpiece. Each jig corresponds to the holes of Type A or B respectively. This will leave much space for adjusting mechanism. Considering the similarity of the two jigs, only the detailed structure of A-jig will be introduced at length in 


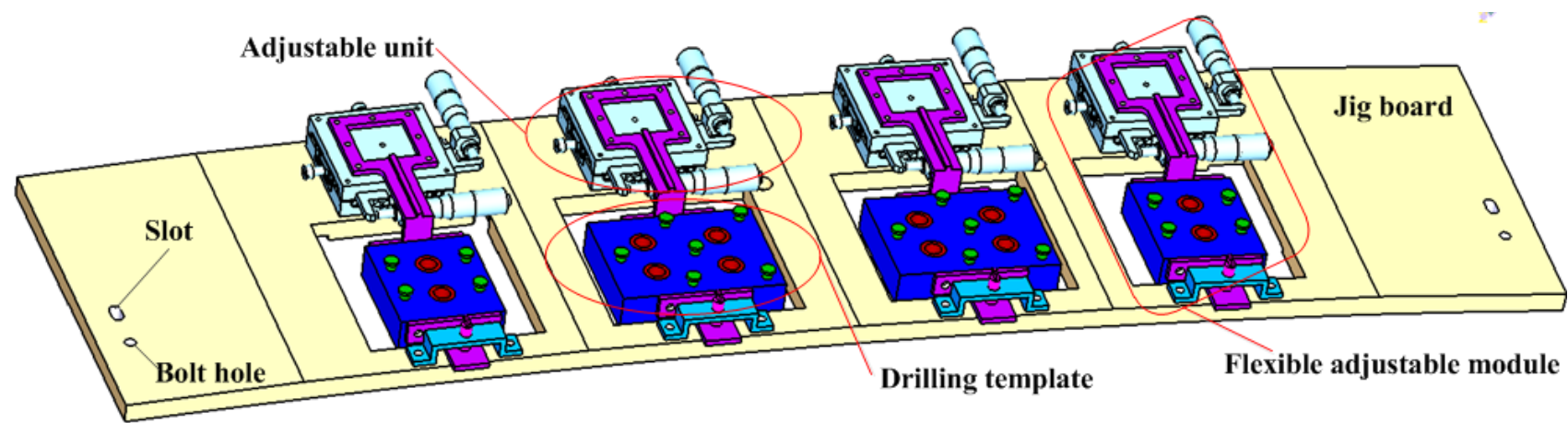

Fig. (2). The 3D model of the flexible adjustable A-jig.

the following test. The detailed 3D model of A-jig is illustrated in Fig. (2).

\subsubsection{The Jig Board}

The A-jig template aimed at Type $\mathrm{A}$ is exampled in Fig. (3), where the bottom of jig board possesses the same ruled surface as the wing-skin it contacts, while the top of the board is milled into four flat planes for installation of flexible adjustable modules. Each plane is mapped from a section of wing-skin which contains a group of two or four holes, so the normal of the board plane coincides with that of the chemical milling plane. This will insure the verticality of the pilot hole in the adjustable module. A rectangular cutout is made in the board to accommodate the real drilling template. The cutout is bigger than the template so that the latter can move around for adjustment in the narrow space. The board is also grooved at the bottom to evade the rows of heads of rivets on the skin. At each end of the jig board, slots and bolt holes are made for locating and clamping the jig on the wing-skin while in service. Specific areas are reserved on the jig for installing and fixing the adjustable modulus. The jig board, designed as a carrier for the adjusting mechanism, is used to fit the curved surface of the wing-skin and insure the global positions and directions of the drilling template.

\subsubsection{The Flexible Adjustable Module}

In A-jig, four flexible adjustable modules are installed on the planes of the jig board. Each module, in charge of a group of holes, contains an adjustable unit and a drilling template connected by a rigid beam between them.

The adjustable unit is a double-deck structure which contains two mobile platforms. The lower platform travels along the guide rails fixed on the board plane in spanwise direction, while the upper platform moves alongside the rail fixed on the lower platform in lateral direction. When they move in separate directions, a micrometer is used to measure the distance they travel. The range in each direction is \pm 10 $\mathrm{mm}$, and the minimum scale is $0.02 \mathrm{~mm}$. The unit also possesses the locking function after proper adjustment.

The small drilling template is specialized for a group of holes with constant relative position on a chemical milling plane for Type-A (or on a T-joint plane for Type-B). The template sinks deep into the rectangular cutout in the board to keep in touch with the wing-skin and fit it. Pilot holes, protected by drill bushings, are made in the template to locate the drilling spots and guide drilling bits. The centerlines of the pilot holes pass through the desired drilling points on the chemical milling plane and coincide with the normal of the points so that the perpendicularity can be insured. Two lock screws are set symmetrical to the center of the pilot hole for clamping positive feed drill motors. The bottom of the template is specialized with scraps discharge and heat discharge grooves. The template is driven by the adjustable unit to move to the right place. The module schematic is illustrated in Fig. (4).

\subsubsection{The Attachments of the Jig System}

Allowing for the requirement that the adjustable module should be locked up once the drilling template finds the right place, a rigid beam connected with the template will be fixed on the jig using wing screws to provide sufficient rigidity to withstand force generated by the associated drilling machines. Besides, the lock bolt will be pinned into a prefabricated notch through the pilot hole and fixed with the mating screw cap to offer extra support. In addition, the adjustable unit also can be fixed to hold the template as mentioned before. Here a $1 / 4$ twist clamping system is used on the lock bolt and the drilling machine to simplify the fixing process.

The flexible adjustable jig is easy to assemble. If a fastener changes size, or one component is damaged, the relevant part can be modified or replaced quickly and it won't be out of service for long. The bushings are easily removable and can be reused therefore provide long term value.

\subsubsection{The Service State of the Jig System}

In service, the jig is hanged on the wing-skin at the bottom of the airfoil, and the adjustable unit drives the drilling template to move to target points. Lock bolts are used to detect preformed holes to locate the drilling position of other holes in each group. Once located, templates can be fixed by the clamping system. Then the positive feed drill motors can be applied to the template by the twist lock to drill connecting holes.

\section{ANALYSIS OF SYSTEMATIC ERRORS FOR FLEXIBLE DRILLING JIG}

\subsection{Analysis of System Error}

As mentioned previously, a chemical milling plane or Tjoint plane will be mated with a curved wing-skin in aircraft 


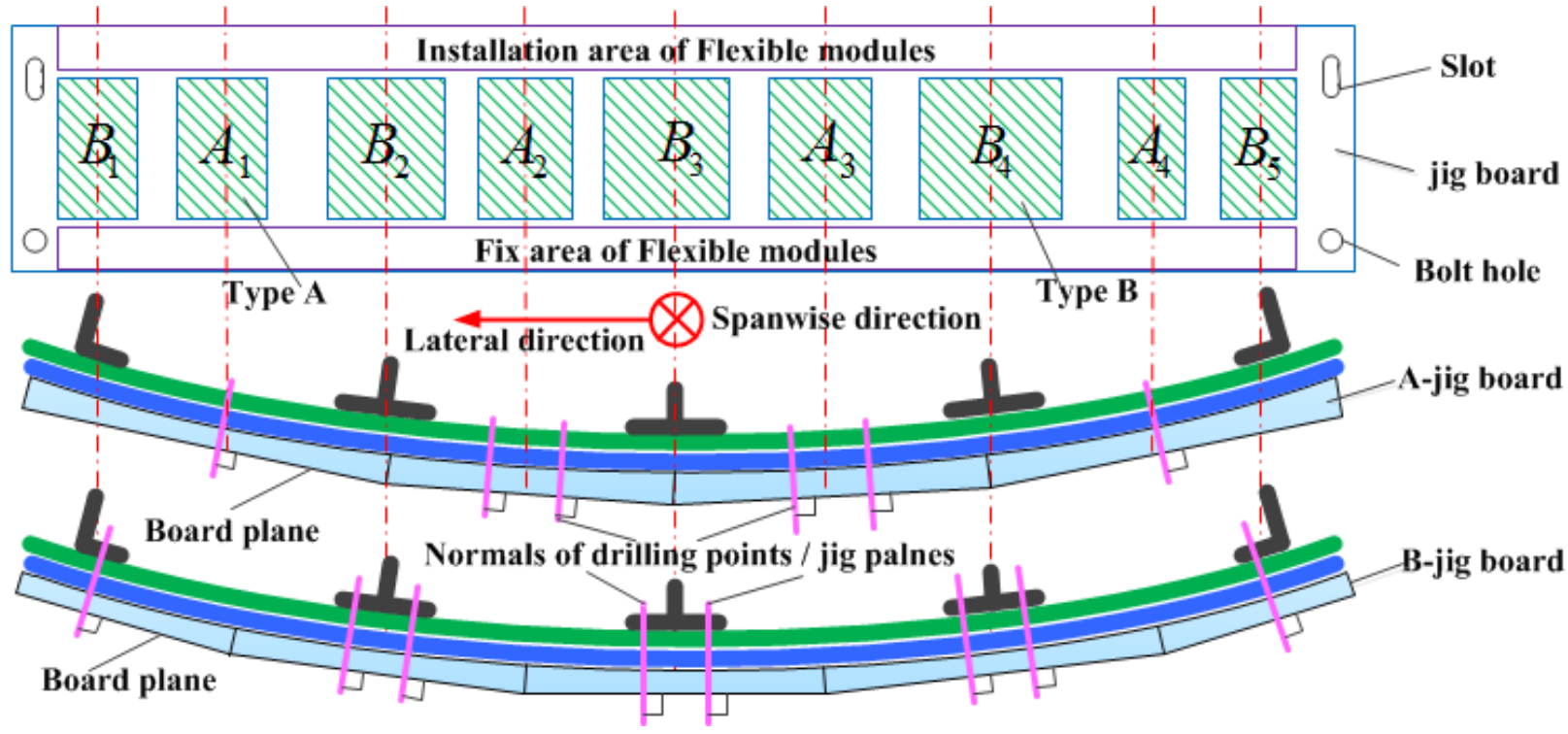

Fig. (3). Division of the groups and the corresponding jig boards.

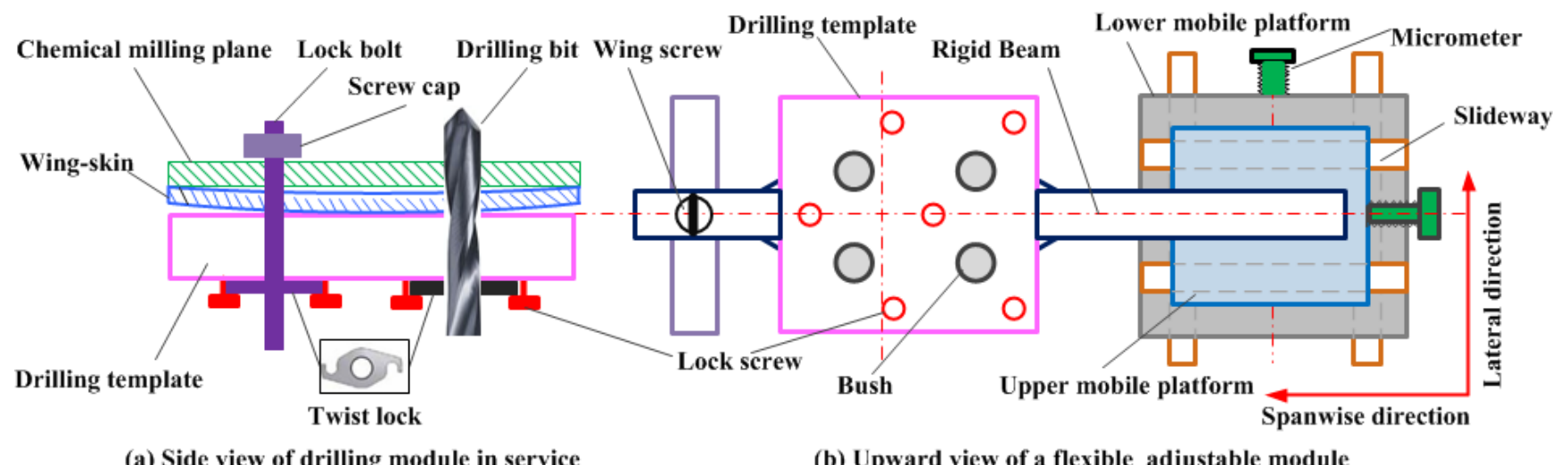

Fig. (4). The schematic flexible adjustable module.

assembly, thus the normal of the drilling point on the wingskin may disagree with that of the mapping point on the milling or T-joint plane. On the assumption that the radius of the curved wing-skin changes smoothly and the skin can be regarded as a planar plane in a small section which covers a group of two or four holes, the milling plane or the T-joint plane is chosen as the datum plane instead of the ruled surface of the skin for the sake of simplicity. So the centerlines of the holes to be drilled are determined by the normal of the milling plane or the T-joint plane, which may deviate from the normal of the wing-skin. Then the systematic error arises.

In spanwise direction, the template plane keeps in contact with the wing-skin along the straight-line generatrix of the ruled skin surface very well. There is no theatrical error in this direction. In lateral direction, there are two situations illustrated in Fig. (4). In the situation of the group with two holes (namely the group of $A_{1}, A_{5}, B_{1}$ and $B_{5}$ in Fig. (3), the hole centers lie in the contact line, in which the normal of template plane coincides with that of the hole center point $M$ on the ruled surface and there is no theatrical errors. While in the situation of group with four holes (the rest of the abovementioned groups ), the holes are some distance away from the contact line $(t / 2$ distance) instead of lying on it, the normal of template plane isn't in accordance with that of the hole center point on the ruled surface. It's more explicit in Fig. (5) that there is a small angle $\theta$ between the drilling direction and the normal direction of hole center point on the wing-skin, which brings about squareness error and position deviation. It should be noted that the errors will also exist when the curved surface is chosen as the reference. In this case, there is nothing but the centerline of the drilling hole deviating from the normal of the milling plane or T-joint plane. So it is an inevitable systematic error.

In the case of the group with four holes, the curve radius $r$ of ruled skin surface in lateral direction changes continuously from $5691.500 \mathrm{~mm}$ to $8095.600 \mathrm{~mm}$. The distance $t$ between two holes in same direction is within the scope of $52.936 \mathrm{~mm}$. The thickness of the connecting area $h$ ranges from $17.810 \mathrm{~mm}$ to $37.570 \mathrm{~mm}$ (actually the 3-layer stackup is much thicker than the 2-layer). According to the geometrical sketch illustrated in Fig. (5), the relationship of the three parameters can be expressed as:

$\sin \theta=t / 2 r$

When the radius $r$ is minimum while the distance $t$ is maximum, the angle $\theta$ gets the highest value, namely: $r_{\min }=5691.500 \mathrm{~mm}$, and $t_{\max }=52.936 \mathrm{~mm}$ respectively. The 


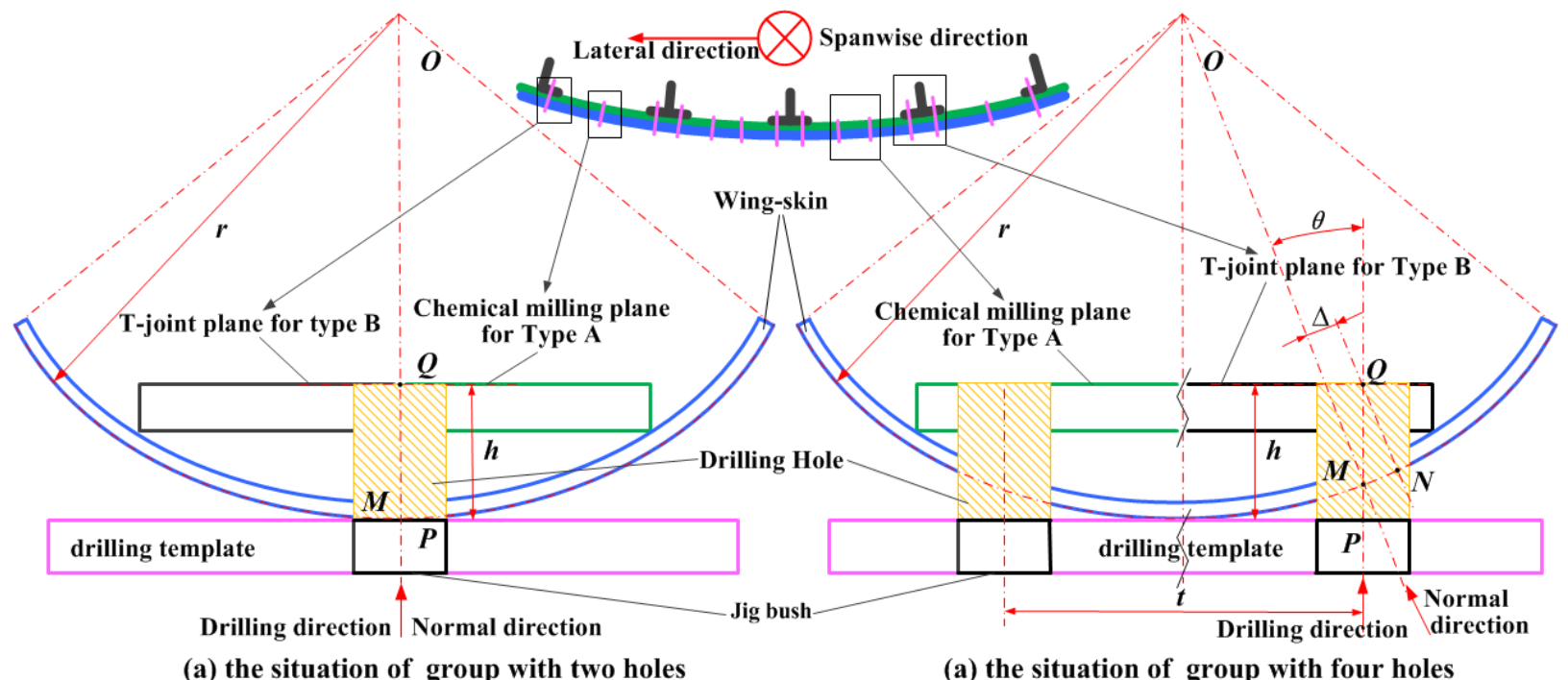

Fig. (5). The origins of system errors.

maximum value of squareness error can be calculated as follows:

$\theta_{\text {max }}=\arcsin \left(t_{\max } / 2 r_{\min }\right)=0.266^{\circ}$

In this condition, the position deviation $\Delta$ can be defined as:

$$
\Delta=|M N| \sin \theta \approx h \sin \theta
$$

where the distance $|M N|$ can be replaced by $h$ since the skin surface possesses a flat curvature and the distance $|M P|$ can be ignored. While both $h$ and $\theta$ are maximum, namely: $h_{\max }=37570 \mathrm{~mm}$, the position deviation $\Delta$ gets the maximum value:

$\Delta_{\text {max }}=h_{\max } \sin \theta_{\text {max }}=0.165 \mathrm{~mm}$

The permitted squareness error is within $0.5^{\circ}$ and the allowable position error ranges from $-0.5 \mathrm{~mm}$ to $1.0 \mathrm{~mm}$ as required in the assembly. In fact, the parameters $r, t$ and $h$ cannot reach their extreme values at the same time, so the actual errors are smaller than the values calculated above, which are within the scope of allowable tolerances. So the flexible adjustable jig system meets the requirements.

\subsection{Analysis of Deformation of Jig Board}

The drilling jig will be hung upside down with the bottom of the board fitting the wing-skin while in service. The jig board will drop and deform to some degree under the gravitation of the adjustable modules and the board of its own. Moreover, the positive feed drill will definitely aggravate the degree. So the error caused by deformation should also be considered apart from systematic errors.

In order to make the drilling jig lightweight, the bulky board is made up of aluminum, which is easily deformed under the gravity of itself and the accessories fixed on it. Since the board is the major origin of deformation for the jig system, the influence of it should be taken into consideration. Here the B-jig is explicitly analyzed as an example as there are five flexible adjustable modules on the board and the deformation is greater than the A-jig.

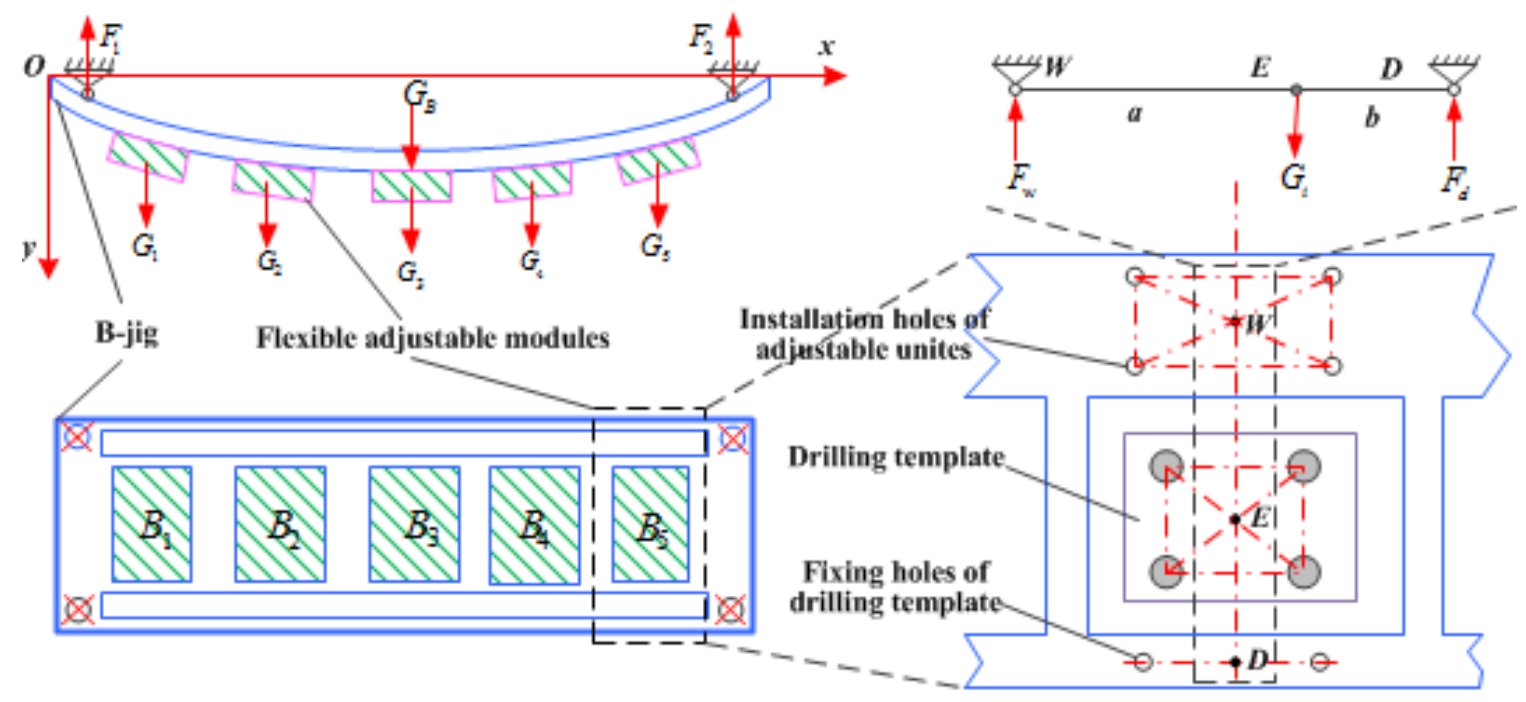

Fig. (6). Force analysis of the B-jig. 
The loading condition of the board is shown in Fig. (6), where the board is fixed on the side edges with slots and bolts and suffers the gravitation of flexible adjustable modules $G_{i}(i=1 \sim 5)$ in vertical direction apart from the selfgravitation. The gravity of adjustable unit is directly applied on the board through four installation holes. The applied force of a template, whose center of gravity is point $E$, is exerted equivalently to the board through the action points $W$ and $P$, the center of the fix and installation holes on the board. When a pneumatic drilling tool is applied on the module, its gravity is also included in $G_{i}$. The reaction of supports from the board is calculated as follows:

$$
F_{w}=\frac{b}{a+b} G_{i}, F_{d}=\frac{a}{a+b} G_{i}
$$

Then the reaction forces $F_{w}$ and $F_{d}$ will be divided and scattered averagely on the fixing and installation hole centers separately for deformation analysis.

A 3D FEA model of the board is developed in the software ABAQUS to simulate the deformation process in service. The model has $1020 \mathrm{~mm}$ in length, $258 \mathrm{~mm}$ in width and $15 \mathrm{~mm}$ in thickness and consists of 683685 nodes and 456138 of 10-node quadratic tetrahedron solid elements. LY12 and Q235 are chosen as the materials of the board for comparison. Apart from self-gravitation, the loads calculated above are applied on the board through installation and fix holes using concentrated force. Remarkably, the displacement of ends of the board needs to be restricted in addition to the fixation of the slots and bolt holes, because the wing-skin confines its movement. The deformation caused by the positive feed unit hang on each drilling template is simulated under the coordinate illustrated in Fig. (6).

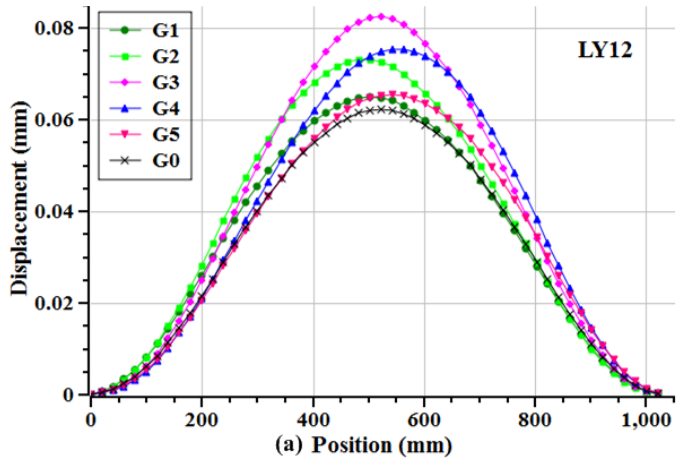

Fig. (7). Deformation analysis of the board: (a) LY12, (b) Q235.

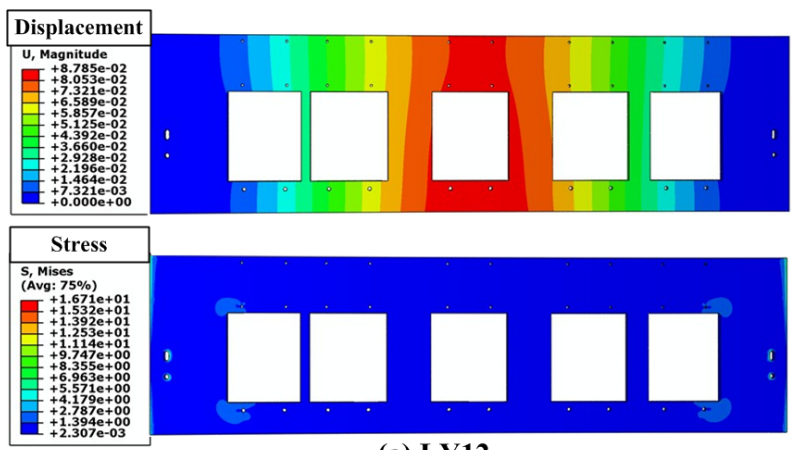

(a) LY12
Fig. (7) shows the magnitude of displacement in each case. Here $G_{i}$ indicates that the positive feed unit is applied on the corresponding position. Especially, $G_{0}$ means the board without the feed unit. It is obviously observed that the two materials follow the same trends except for the different magnitude caused by distinct properties. The deformation degree is the least when there is no feed unit hung on the board. As the feed unit moves from the either end to the center workpiece, the magnitude increases until it reaches the peak value at position $G_{3}$. The middle part of the board has much heavier deformation than the other parts while the feed unit slightly affects the position of the peak value.

In whichever cases, the deformation magnitude is no more than $0.082 \mathrm{~mm}$ for LY12 and $0.044 \mathrm{~mm}$ for Q235 respectively, and the stress level is far from yield for both materials as Fig. (8) illustrates. The deformation and stress levels are within the permissive scope, which indicates that the design is acceptable. When chosen to fabricate the jig board, LY12 will be much lighter than Q235, while the latter can provide greater rigidity than the former.

\section{APPLICATION PROCEDURES OF THE FLEXIBLE DRILLING JIG}

The flexible drilling jig system is applied to the aircraft assembly through following steps:

1) Put the parts to be assembled together in the right position and make the mating interfaces clamped tightly.

2) Drill the preformed holes, which are much smaller than the final holes, in each group for locating the jig boards and drilling templates.
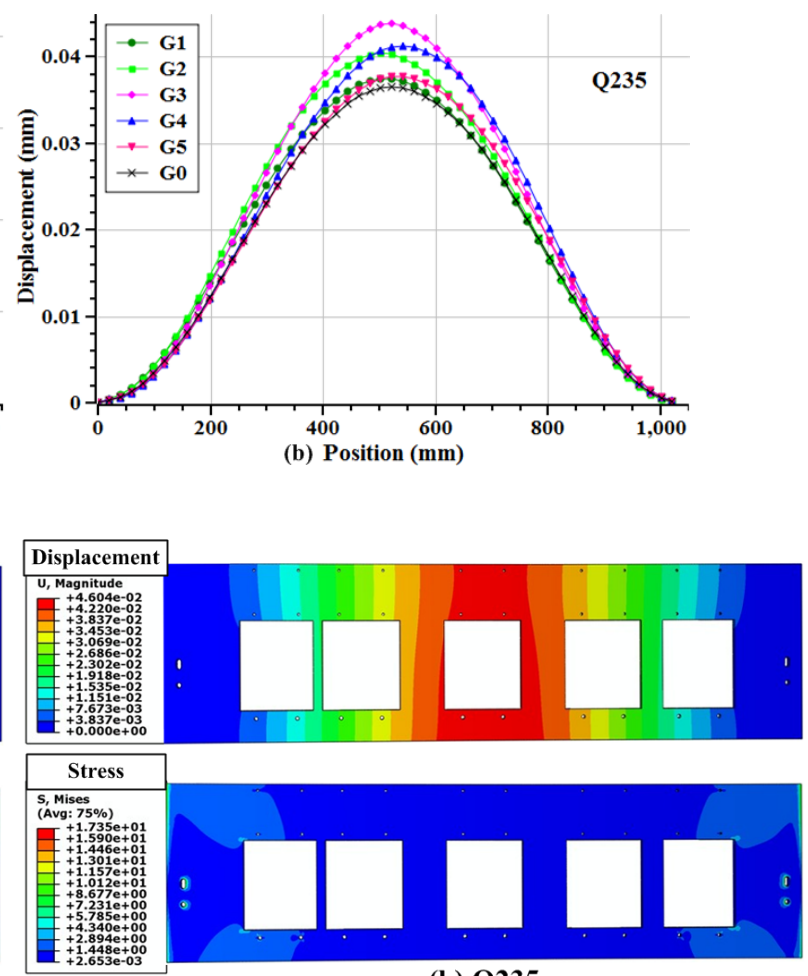

(b) Q235

Fig. (8). Displacement and stress nephrogram in case G3: (a) LY12, (b) Q235. 
3) Secure the jig to the workpiece through slot holes and bolt holes.

4) Insert the bolt screws into the pilot holes and adjust the drilling templates to make the bolts pass through the preformed holes to secure the right position. Then twist the bolt screws and wing screw tightly, and fix the adjustable units.

5) Move the machine with the $1 / 4$ turn clamping system up against the jig template and lock it manually by completing the corresponding rotary movement. Once the clamping system is engaged, the drilling process can be started.

6) Exchange the work stations of locks crews and the drill machine, and reaming the preform holes to the final state.

7) Remove the jig and clear the scraps around holes.

\section{CONCLUSION}

This paper presented a flexible drilling jig system which is adjustable for wing-fuselage connection. The paper mainly carried out the following work:

1) The general configuration of the assembly problem is introduced.

2) The design idea is analyzed and the components of the flexible drilling jig are comprehensively presented with illustrations.

3) The analysis of system errors (square errors and position deviations) and deformation levels with or without positive feed unit in service are carried out to validate the utility of the drilling jig.

4) The application procedure of the flexible drilling jig is taught in the end.

The flexible drilling jig system offers a cost-effective solution to the drilling of accurate holes, normal or curved structures. When compared with traditional tooling associated with this objective, the flexible drilling jig offers a number of benefits. It not only reduces the design, manufacture and costs, but also ensures the assembly precision.

\section{CONFLICT OF INTEREST}

The authors confirm that this article content has no conflict of interest.

\section{ACKNOWLEDGEMENTS}

The authors gratefully acknowledge the support of Fund of National Engineering, Research Center for Commercial Aircraft Manufacturing, China (Program No. SAMC13-JS15-029), National Natural Science Foundation of China (Grant No. 51275410), and Aviation Science Foundation Project (Program No. 2014ZE53056). The authors are also grateful to the editors and the anonymous reviewers for helpful comments.

\section{REFERENCES}

[1] H. Yuan, "Manufacturing technology of connecting hole in aircraft structures," Aeronautical Manufacturing Technology, vol. 1, pp. 96-99, 2007.

[2] E. Oberg, F. Jones, H. Horton, H. Ryffel, C. McCauley, Eds., Machinery's Handbook, Industrial Press Inc., New York 2000.

[3] D.W. Robinson, "Practical Design and Use of Drill Template Jigs in Spacecraft Manufacturing," In: ASME 2003 International Mechanical Engineering Congress and Exposition. American Society of Mechanical Engineers, 2003, pp. 251-258.

[4] N. Jayaweera, O.J. Bakker, T. Smith, A. Popov, S. Ratchev, and A. Turnock, "Flexible tooling for wing box rib clamping and drilling," SAE International Journal of Aerospace, vol. 4, pp. 1048-1056, 2011.

[5] A. Wiemann, and S. Leverington, "Flexible template for drill feed units," SAE International Journal of Aerospace, 2007, pp. 63-66.

[6] T.K. Lostlen, “Adjustable Drill Jig”, U.S. Patent No.5807036A. Washington DC: U.S. Patent and Trademark Office, 1998. 